\title{
Dynamic and Progressive Control of DNA Origami Conformation by Modulating DNA Helicity with Chemical Adducts
}

Haorong Chen, ${ }^{\dagger}$ Hanyu Zhang, ${ }^{\dagger}$ Jing Pan, ${ }^{\dagger}$ Tae-Gon Cha, ${ }^{\dagger}$ Shiming Li, ${ }^{\ddagger}$ Joakim Andréasson, ${ }^{\ddagger}$ and Jong Hyun Choi ${ }^{*} \dagger$

${ }^{\dagger}$ School of Mechanical Engineering, Purdue University, West Lafayette, Indiana 47907, United States

${ }^{\ddagger}$ Department of Chemistry and Chemical Engineering, Chalmers University of Technology, SE-412 96 Gothenburg, Sweden

\section{Supporting Information}

ABSTRACT: DNA origami has received enormous attention for its ability to program complex nanostructures with a few nanometer precision. Dynamic origami structures that change conformation in response to environmental cues or external signals hold great promises in sensing and actuation at the nanoscale. The reconfiguration mechanism of existing dynamic origami structures is mostly limited to single-stranded hinges and relies almost exclusively on DNA hybridization or strand displacement. Here, we show an alternative approach by demonstrating on-demand conformation changes with DNA-binding molecules, which intercalate between base pairs and unwind DNA double helices. The unwinding effect modulates the helicity mismatch in DNA origami, which significantly influences the internal stress and the global
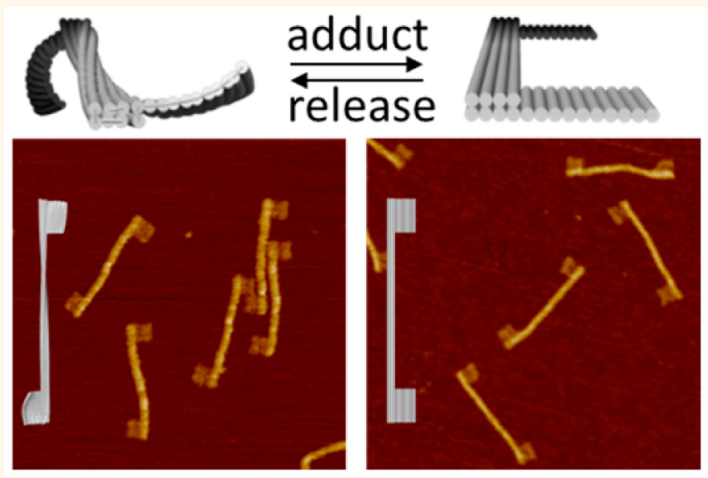
conformation of the origami structure. We demonstrate the switching of a polymerized origami nanoribbon between different twisting states and a well-constrained torsional deformation in a monomeric origami shaft. The structural transformation is shown to be reversible, and binding isotherms confirm the reconfiguration mechanism. This approach provides a rapid and reversible means to change DNA origami conformation, which can be used for dynamic and progressive control at the nanoscale.

KEYWORDS: DNA origami, DNA binding, conformation change

$\mathrm{I}$ $\mathrm{n}$ addition to their crucial biological functions, nucleic acids can also serve as information-rich materials that selfassemble with nanometer precision. ${ }^{1}$ A single RNA strand can fold itself into a complex conformation. ${ }^{2}$ Through rational sequence designs ${ }^{3}$ and special chemical linkers, ${ }^{4,5}$ various intricate $2 \mathrm{D}$ and $3 \mathrm{D}$ structures have been demonstrated. DNA origami has garnered great attention because it facilitates unprecedented assembly complexity. ${ }^{6}$ The versatile programmability, coupled with a variety of available chemical modifications, ${ }^{7,8}$ made DNA origami a valuable research tool and spawned many emerging applications. As static templates, DNA origami has been used to organize proteins ${ }^{9-11}$ and nanoparticles ${ }^{12,13}$ or to serve as lithographic masks to fabricate other materials. ${ }^{14-17}$ Dynamic origami structures change their conformation in response to environmental cues or external signals. They complement the impressive spatial resolution with structural controllability and thus promise rich functionalities in sensing and actuation. For example, a pincer origami can pinch close upon binding to molecular targets, ${ }^{18}$ and a clam-like switch can open up and expose payloads upon recognizing a combination of physiological cues. ${ }^{19}$

Dynamic DNA origami structures demonstrated to date are limited in terms of their reconfiguration mechanisms. The reconfigurable structures are mostly made of single-stranded (ss) hinges that connect two rigid double-stranded (ds) domains, and their shape change can usually be summarized as a "close/open" process; the structure closes when linking ligands (e.g., ss-DNA linkers) bind the two moving domains together and opens when the linking ligands are disengaged (e.g., via strand displacement).$^{20,21}$ Besides hybridization and strand displacement, which allow complex logical controls, ${ }^{22}$ linking ligands can also be rendered responsive to light, ${ }^{23,24}$ thermal, ${ }^{25}$ or biological signals. ${ }^{19}$ Despite their versatile controllability, such ss-hinge-based mechanisms are not without drawbacks. First, the origami conformation is not well-defined

Received: February 22, 2016

Accepted: April 8, 2016

Published: April 8, 2016 


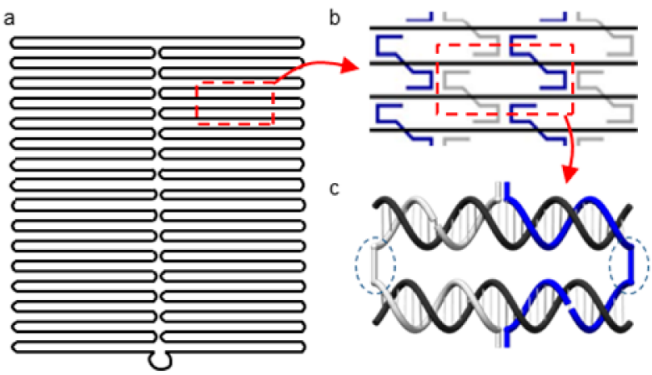

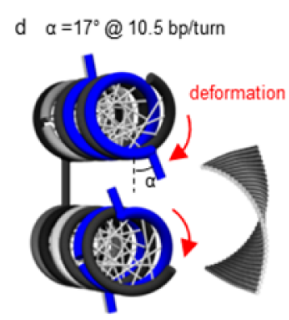

under-compensated

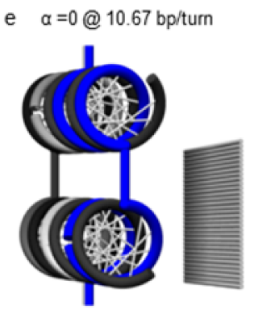

compensated

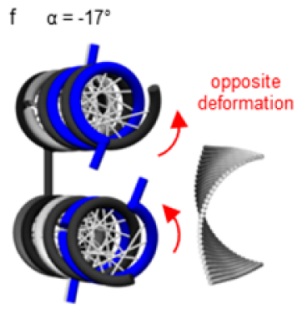

over-compensated

increasing belicál pitch / adduct concentration
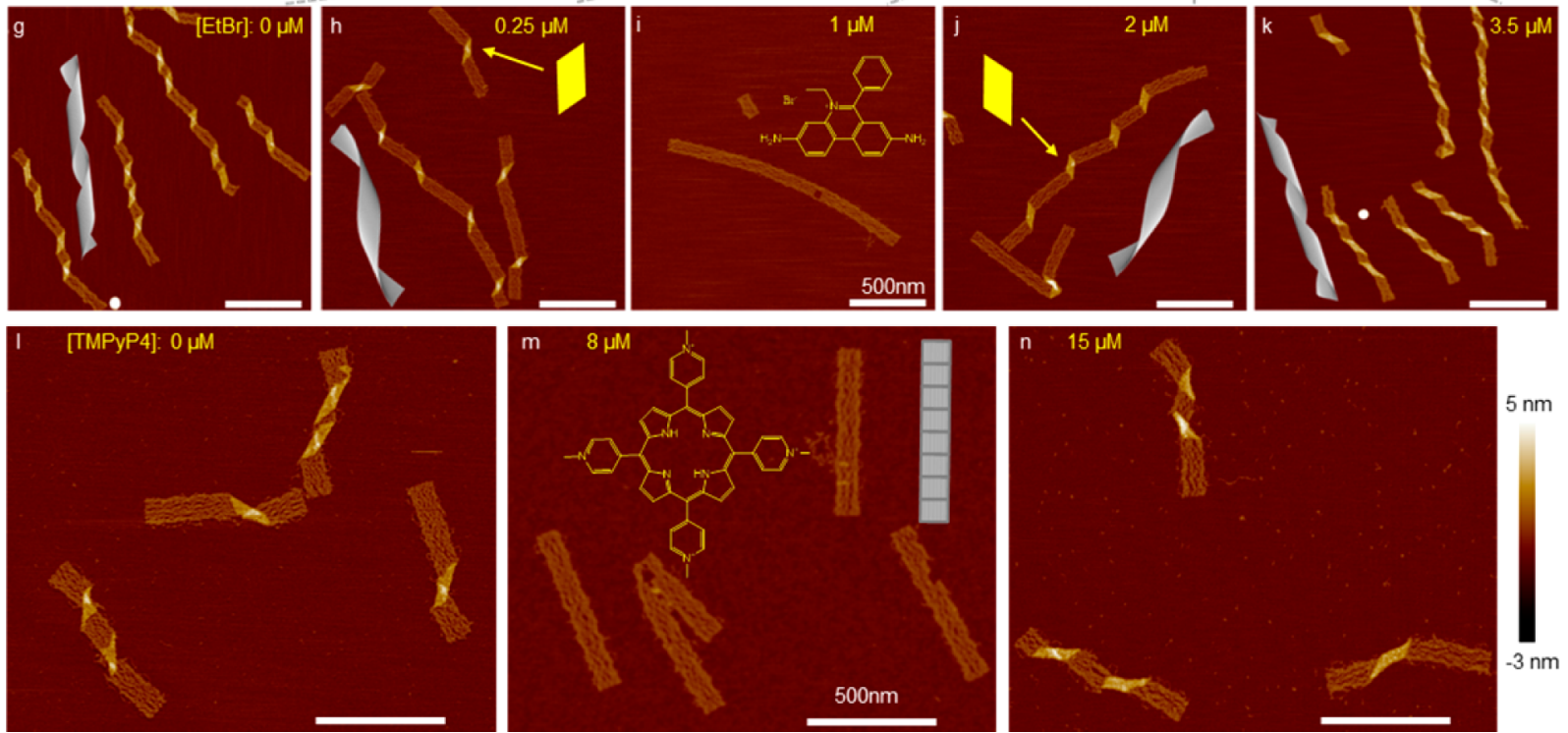

Figure 1. Conformation change in origami tiles and polymerized ribbons. (a) Scaffold folding path of the 32-helix rectangular origami. (b) Schematic of a part of the origami tile demonstrating the periodic arrangement of the staples (blue and gray) against the scaffold (black). (c) Schematic of the unit motif of the origami tile. The staples (blue and gray) pair with the scaffold (black) into double helices connected by crossovers (circled). (d-f) Side views of the unit motif in (c), with corresponding simulated origami conformations on the right. Under intrinsic conditions, the double helices twist more than needed for forming a crossover connection. The mismatch ultimately leads to the global curvature in the origami tile (d). At an appropriate intercalation density, the helicity mismatch is compensated and crossover forms without distortion. Correspondingly, the tile assumes a planar conformation (e). Excessive intercalation overcompensates the helicity mismatch and leads to the opposite curvature of the origami tile $(\mathrm{f}) .(\mathrm{g}-\mathrm{k})$ AFM images of polymerized origami ribbons under various EtBr concentrations, with the ribbon concentration kept constant at $2 \mathrm{nM}$. Corresponding equilibrium conformations are simulated with helical pitches of 10.50 and $10.59 \mathrm{bp} /$ turn and shown in the insets of $(\mathrm{g})$ and $(\mathrm{i})$. The ribbon handedness can be determined from the shape of the parrallelogram kinks, as highlighed in $(\mathrm{h})$ and $(\mathrm{j})$. As the EtBr concentration increases, the right-handed kinks reduce in density until complete disappearance; then left-handed kinks appear and increase in density. (1-n) AFM images of size-controlled 8-mer ribbons as a function of TMPyP4 cocnentration. Right-handed kinks initially disappear, to be replaced by left-handed kinks with increasing TMPyP4 concentration.

in the open state because the two parts are hanging on soft sshinges and are subject to large conformation fluctuation. Second, the close/open process switches between two drastically different states and, therefore, does not allow the progressive fine-tuning of conformation. Deformable ds-joints are promising alternatives to ss-hinges for overcoming these drawbacks. Compared with ss-DNA, ds-DNA is 2 orders of magnitude stiffer. ${ }^{26}$ As a result, the dynamic origami structure is much less susceptible to thermal fluctuation and better at maintaining its defined conformation. While ss-hinges behave like free joints, compliant ds-domains behave like spring joints, ${ }^{27,28}$ which adjust their geometry continuously in response to changing stresses. Based on this principle, progressive conformation changes of DNA origami can be achieved by modulating their stress in fine steps. ${ }^{28}$

To exploit the advantage of ds-joints for dynamic DNA origami, a chemical strategy is needed to modulate the internal stress and supply the considerable elastic energy associated with the deformation of the ds-domains. In this work, we demonstrate that the noncovalent binding of DNA intercalators, such as ethidium bromide (EtBr), meso-tetra $(\mathrm{N}$-methyl-4pyridyl)porphine (TMPyP4), and other DNA-binding molecules, can serve as such energy sources. Intercalation unwinds DNA duplexes ${ }^{29}$ and, thereby, modulates the helicity mismatch. This effect has been confirmed to significantly affect the internal stress of DNA origami ${ }^{30}$ and shown to induce global conformation changes. ${ }^{31}$ Compared to other methods that modulate the stress in DNA origami by modifying the in silico design, ${ }^{28,32}$ intercalation facilitates the on-demand control over origami conformation. Here, we demonstrate the effectiveness of this experimentally simple approach in dynamic structural fine-tuning with two model origami systems: (1) hierarchically assembled origami nanoribbons and (2) monomeric origami shafts. 


\section{RESULTS AND DISCUSSION}

Conformation Change of Single-Layer Origami. To illustrate the mechanism, we first used a rectangular DNA origami tile (Figure 1a), whose staple strands (blue and gray in Figure $1 b$ ) are arranged in a periodic array and form crossovers every 32 base pairs (bp). Except for altered connectivity at the boundaries, the tile can be treated as a periodic lattice propagated by a unit motif (boxed in Figure $1 \mathrm{~b}$ and illustrated in Figure 1c). The motif consists of two $32 \mathrm{bp} \mathrm{B-form} \mathrm{double}$ helices connected by crossovers on both ends. Ideally, the double helices should twist exactly three full turns (corresponding to $10.67 \mathrm{bp} /$ turn helical pitch) in order for both pairs of crossover points to be oriented correctly to facilitate crossover connection and origami formation. However, since the intrinsic helical pitch of B-form DNA is $\sim 10.5 \mathrm{bp} / \mathrm{turn},{ }^{6,32-34}$ the $32 \mathrm{bp}$ double helices twist $\alpha=\sim 17^{\circ}$ more than designed in their equilibrium state, where $\alpha$ represents the mismatch angle defined as the angle that a $32 \mathrm{bp}$ double helix overtwists in its most relaxed state (Figure 1d; see Supporting Information for detailed calculation of $\alpha$ from the helical pitch). The overtwist leads to misalignment between the crossover points and necessitates the distortion of the double helices from their relaxed conformation. The distorted helices exert right-handed torques to neighboring helices, which collectively cause a global deformation of the origami from its designed planar conformation, as shown by the simulation results ${ }^{34}$ (insets of Figure 1d).

The helical twist mismatch causes the origami conformation to deviate from its in silico design. Such an effect has been observed in the past and is often unwelcomed. ${ }^{6,35}$ Several strategies have been developed to avoid such effects by minimizing the mismatch with design modifications ${ }^{33}$ or by introducing additional compensating stresses. ${ }^{36}$ Such effects, however, can also be actively exploited to create twisted and curved static structures through deliberate insertion and/or deletion of base pairs between crossovers during in silico origami design. ${ }^{32}$ In this work, we take the opposite approach by modulating the mismatch through tuning the relaxed helicity of B-form DNA. DNA intercalators such as EtBr can insert between the stacks of planar base pairs and unwind the double helix (reduce the helical twist). This effect can be used to compensate the overtwist of double helices in origami. At an appropriate intercalation density, the overtwist may be completely compensated ( $\alpha$ reduced to 0 , Figure 1e). Since the crossover points are naturally aligned, no further distortion is needed and the origami assumes its designed conformation. When intercalators are bound in a higher density, the mismatch will be overcompensated $(\alpha<0)$, and the double helices will be distorted in the opposite direction, resulting in the opposite curvature of the origami (Figure 1f).

Atomic force microscopy (AFM) was used to visualize the change of origami conformation under different intercalator concentrations. It is worth noting that, as in most highresolution imaging methods, AFM requires the sample firmly fixed onto an imaging substrate. The deposited origami typically collapses and flattens to maximize their contact area with the substrate. To unambiguously demonstrate the conformation change, the curvature of individual tiles should be accumulated by polymerizing the tiles into elongated nanoribbons with a set of linker strands (see Figure S1). Figure $1 \mathrm{~g}-\mathrm{k}$ shows the typical AFM images of origami ribbons under various $\mathrm{EtBr}$ concentrations, along with corresponding
$\mathrm{CanDo}^{34}$ simulation results. Under intrinsic conditions (i.e., no $\mathrm{EtBr}$ ), the ribbons exhibited dense kinks in the form of parallelogram-shaped double-layer regions (Figure 1g).

Such kinks indicate a right-handed spiral conformation of the ribbons in solution (Figures S3 and S4; also see detailed discussion and experimental validation in Supporting Information). At a low concentration of $\operatorname{EtBr}($ e.g., $0.25 \mu \mathrm{M})$, significant reduction of the kink density was observed (Figure 1h), confirming the partial compensation of helicity mismatch and the reduction of the right-handed global twist. At appropriate EtBr concentration $(\sim 1 \mu \mathrm{M}$ in this case), the kinks disappeared completely, even for very long ribbons, corresponding to the perfect compensation of the twist mismatch (Figure 1i). Further increase of EtBr concentration caused the appearance of kinks that display the opposite handedness. As highlighted in Figure $1 \mathrm{~h}, \mathrm{j}$, the parallelogram shapes are mirror images of each other, indicating reversed handedness when helicity mismatch is overcompensated. At an even higher EtBr concentration (3.5 $\mu \mathrm{M})$, the kink density increased further, as expected for increased degree of overcompensation. Statistical analysis of the kink density clearly demonstrates the systematic progression of origami morphology and the conformational effect of EtBr (Figure S12).

Similar disappearance and reappearance of kinks may also be triggered by other intercalators such as TMPyP4 (Figure S13). This is demonstrated with length-controlled 8-mer ribbons assembled from the monomer tiles (Figure $11-\mathrm{n}$ and Figure S14). The kinetics of the conformation change were found to be quite fast. Expected morphology was always observed with incubation time as short as $10 \mathrm{~min}$, the time scale needed for AFM deposition. Therefore, we conclude that the reconfiguration completes in a time scale of $10 \mathrm{~min}$ or less, which is comparable with or faster than the kinetics of other dynamic DNA origami based on strand displacement or hybridization. $^{21,37}$

The conformation change can also be demonstrated by the change of cyclization kinetics of the monomer tiles. Similar to a compliant linear polymer, which can bend over and then be ligated into a circular form, ${ }^{38}$ the rectangular tile can be cyclized into a short cylinder by linker strands that simultaneously hybridize with two opposite edges of the tile (Figure S5a,b). ${ }^{37}$ The kinetics of such cyclization reactions are sensitive to the stiffness and conformation of the origami tile. The stiffer the structure, the harder it is to bend over and cyclize. Conformation-wise, the curvature of the tile induced by the helicity mismatch has been reported to prevent the proper alignment of the two edges to be ligated and, thus, hinders the cyclization kinetics. ${ }^{37}$ As shown in Figure 1, intercalators can compensate the helicity mismatch by reducing the global curvature, hence promoting the cyclization reaction. Excess intercalators would instead overcompensate, induce the opposite curvature, and should cause the cyclization to slow down again. We measured the cyclization yield as a function of binder (EtBr and TMPyP4) concentrations and confirmed the expected kinetic trend: the reaction rate increased with increasing intercalator concentration until the tiles were approximately flattened. Further increase of intercalator concentration was accompanied by a steep decrease of cyclization rate (Figure S5f,g), which suggests that, besides the unwinding effect, intercalation also causes stiffening of DNA double helices (see detailed discussion in Supporting Information). ${ }^{39,40}$ 
Linearly Controllable Torsion of an Origami Shaft. In an origami designed with constant crossover spacing and high symmetry, the uniform torque induced by helicity mismatch should intuitively cause only torsion in the global conformation. The origami ribbon assumes a spiral conformation because its single-layered nature allows the nonlinear coupling between twisting, bending, and stretching deformation modes. ${ }^{41}$ In an origami with increased number of layers, such coupling is discouraged, and purely torsional deformation can be obtained.

To demonstrate the torsional deformation, we designed an origami shaft made of a stem with two small single-layered flags attached to both ends (Figure 2a,b). The stem consists of eight
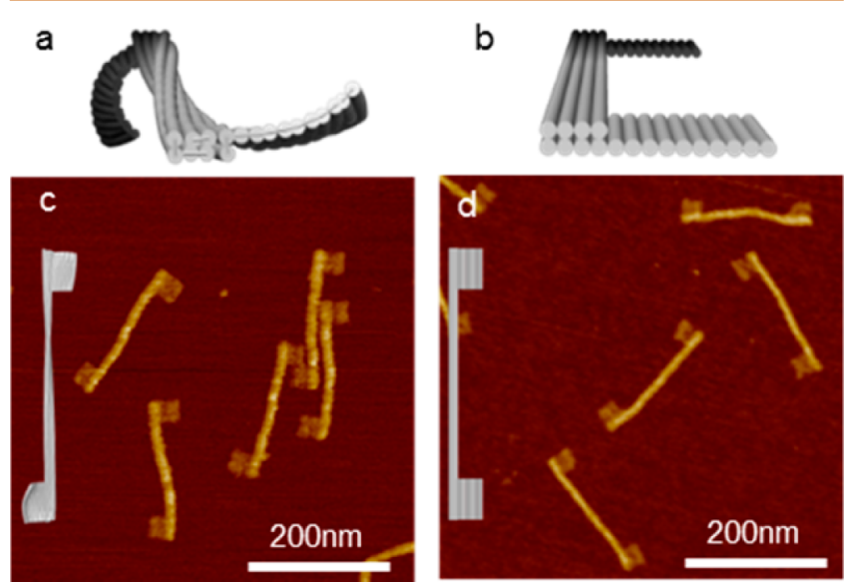

Figure 2. Conformation change of the origami shaft. (a) Simulated shaft conformation under intrinsic conditions $(10.5 \mathrm{bp} /$ turn helical pitch). (b) Designed shaft conformation (at $10.67 \mathrm{bp} /$ turn). (c,d) AFM images of the origami shafts with the EtBr concentration at 0 (c) and $0.75 \mu \mathrm{M}(\mathrm{d})$. The shafts are predominantly in trans (c) and cis (d) conformations.

closely packed DNA double helices. The two flags were intended to display the torsional twist of the stem, which can be quantitatively described by the angle of rotation $(\theta)$ between the two ends. In the designed conformation, $\theta$ is 0 and the two flags are on the same side of the stem (cis-form, Figure $2 b$ ). Similar to the origami tile, the shaft was also based on square lattice, ${ }^{42}$ with a designed helical pitch of $10.67 \mathrm{bp} /$ turn. Under intrinsic conditions ( $10.5 \mathrm{bp} / \mathrm{turn}), \mathrm{CanDo}^{34}$ simulation predicts that the stem assumes a right-handed half turn. Therefore, $\theta$ is $\sim 180^{\circ}$, and the two flags are sent to opposite sides (trans-form, Figure 2a). The simulation results were confirmed by AFM imaging. In the absence of binders (Figure $2 \mathrm{c})$, the shafts were predominantly observed in the trans-form, whereas cis domination could be triggered by addition of $\mathrm{EtBr}$ (Figure 2d). CanDo simulation also predicts that the stems maintain their straight global conformation (Figure 2a,b) under moderate helicity mismatches, and that $\theta$ is proportional to the mismatch angle $\alpha$ (Figure S8). Since intercalators primarily unwind DNA double helices, ${ }^{43}$ the simulation results confirm the feasibility of inducing pure torsion via intercalation. As in the origami ribbon case, binders are expected to initially compensate the helicity mismatch and twist the shaft gradually from trans- to cis-form. Excess intercalators should overcompensate the mismatch and induce a left-handed twist which rotates the two flags to opposite sides again.

Experimentally, the origami shaft samples were deposited for AFM imaging under various EtBr concentrations. Due to the flattening effect of AFM, the well-formed origami shafts were observed in either trans- or cis-form. A systematic shift of the trans/cis statistical distribution was observed (Figure 3a). The
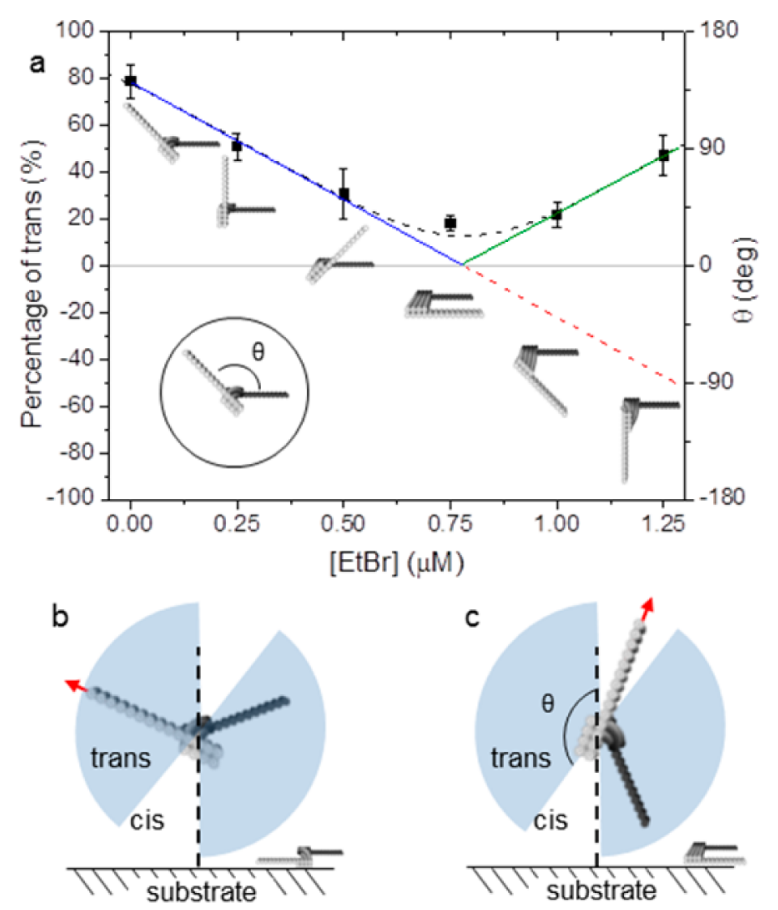

Figure 3. (a) Statistically determined trans/cis distribution as a function of EtBr concentration. The data points largely follow a vshape (blue and green line). The red line is the mirror image of the green line and demonstrates that the blue and green lines have the same slope. The twist angle of the shaft can be described by the angle $\theta$ between the two flags (shown in the inset). By assuming that $\theta$ decreases linearly with $\mathrm{EtBr}$ concentration (following the blue and red line), the v-shape trend can be explained. By considering thermal fluctuation, a more accurate prediction can be made (represented by the dashed black curve), which accounts for all the data points. $(b, c)$ Schematics illustrating how a shaft can be deposited into either trans or cis morphology. When the shaft orientation (represented by the red arrow) is within the blueshaded area, the two flags are on the opposite side of the surface normal (black dashed line), and the shaft is deposited into transform (b). Conversely, cis-form is deposited (c). The probability for the trans-form is proportional to the shaded area and equals $|\theta|$ / $180^{\circ}$.

percentage of trans-form could be well-described by a linear function that initially decreases with increasing $\mathrm{EtBr}$ amount and then increases with the same slope. Such a linear trend strongly suggests a linear dependence of stem torsion angle $\theta$ on $\mathrm{EtBr}$ concentration (vide infra).

The morphology of an origami shaft observed by AFM is determined by the conformation it assumes after deposition. To understand the experimental trend of trans/cis percentage, it is necessary to analyze the deposition process. Figure $3 b, c$ illustrates a simple model which assumes that the stem of the shaft remains parallel to the substrate while it approaches the surface. The deposited conformation is likely determined by the transient orientations of the flags. If the flags are on the opposite side of the surface normal (shown as the dashed line), the two flags will be pulled down to the surface on either side of the stem, and the shaft will be trapped in trans-form (Figure 3b). Conversely, the shaft will be deposited into cis-form (Figure 3c). A simple geometric analysis shows that the two 

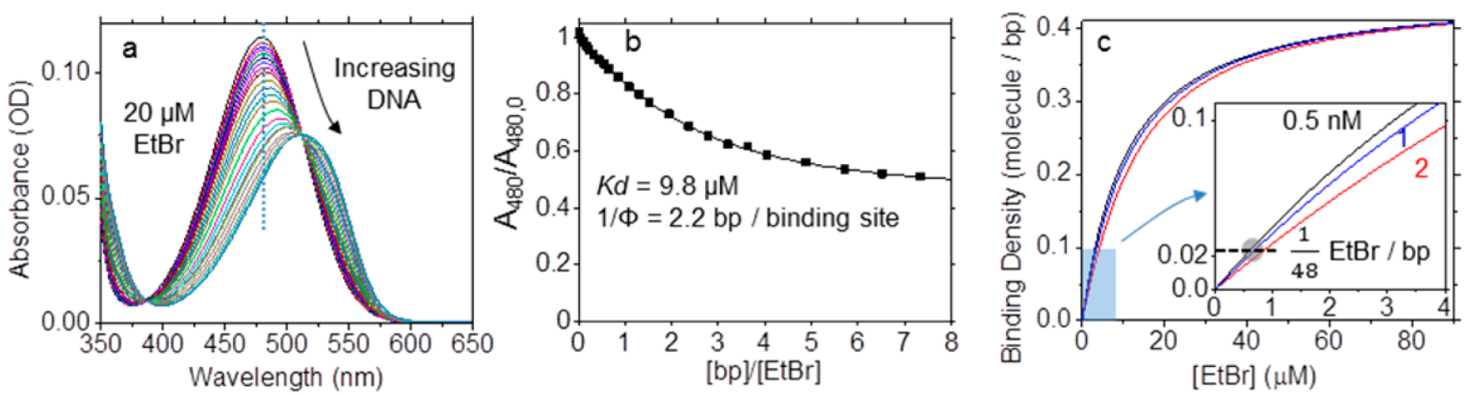

Figure 4. Binding isotherm by absorbance titration. (a) Absorption spectra of mixtures of EtBr and monomeric origami tiles. EtBr concentration was kept at $20 \mu \mathrm{M}$. (b) Absorbance at the original peak position at $480 \mathrm{~nm}$, indicated by the dotted blue line in (a), as a function of $\mathrm{DNA} / \mathrm{EtBr}$ ratio. The data points coincide with the curve from a nonlinear least-squares fit. The extracted binding parameters, dissociation constant $K_{\mathrm{d}}$ and maximum binding density $\Phi$, are displayed in the panel. (c) Calculated EtBr binding density plots for three origami monomer concentrations $(0.5,1$, and $2 \mathrm{nM}$ in black, blue, and red, respectively) as a function of EtBr concentration. When the binding density is smaller than 0.1 molecule/bp (blue-shaded area), which is the case in our experiment, the binding density is approximately proportional to $\mathrm{EtBr}$ concentration. To compensate the twist mismatch in our model origami systems, the required EtBr intercalation density was calculated to be $1 / 48$. The EtBr concentration for achieving such density can be read from the inset and matches the experimentally determined compensating concentrations (gray circle).

flags will be placed on opposite sides of the surface normal when the shaft orientation (represented by the red arrow) is within a certain range (shown as the shaded sector). The sector angle of the shaded area equals $|\theta|$. Since the transient shaft orientation is likely randomized, the probability for deposition as trans-form can be expressed as $|\theta| / 180\left(-180^{\circ} \leq \theta \leq 180^{\circ}\right)$. This function naturally explains the v-shaped trend, assuming that $\theta$ decreases linearly from $\sim 145$ to $\sim-90^{\circ}$ when $\mathrm{EtBr}$ concentration increases from 0 to $1.25 \mu \mathrm{M}$.

Thermal fluctuation needs to be considered for a more comprehensive and quantitative understanding of the observed population shift. The torsional stiffness of similar DNA origami stems was previously measured by magnetic tweezers, ${ }^{27}$ from which we estimate the torsional persistence length of $800 \mathrm{~nm}$ for our shaft. Due to the finite stiffness, transient torsion angle $\theta$ can fluctuate about the equilibrium value $\theta_{\mathrm{o}}$. Under thermal equilibrium, $\theta$ should follow the Maxwell-Boltzmann distribution. Since the fluctuation increases the elastic energy of the shaft by $1 / 2 K_{\mathrm{t}}\left(\theta-\theta_{\mathrm{o}}\right)^{2}$, where $K_{\mathrm{t}}$ is the torsional spring constant of the stem, we can deduce that $\theta$ follows a normal distribution: $p(\theta)=\frac{1}{\sqrt{\pi} \sigma \theta} \exp \left\{-\frac{\left(\theta-\theta_{0}\right)^{2}}{(\sigma \theta)^{2}}\right\}$, where $\sigma \theta\left(40.5^{\circ}\right)$ characterizes the magnitude of thermal fluctuation (see Supporting Information for detailed calculations). When $\theta_{\mathrm{o}}=$ 0 , any fluctuation will increase $|\theta|$ and contribute probability of trans deposition. Integration over all the transient conformations predicts that the probability for trans-form is $\sqrt{\frac{1}{\pi}} \cdot \frac{\sigma \theta}{180}=14 \%$, which is close to the minimum value we observe. Similarly, thermal fluctuation at $\theta_{\mathrm{o}}= \pm 180^{\circ}$ should limit the trans percentage to less than $86 \%$. The reasonable agreement of the experimentally observed trend and our model strongly indicates that the torsion of the shaft is indeed linearly controllable by intercalator concentration.

Binding Isotherm and Quantitative Understanding of Intercalation Density. To elucidate the relationship between intercalator concentration, intercalation density, and the degree of induced origami conformation change, we studied the binding isotherm between intercalator and DNA origami. Absorption spectra were monitored, while DNA was titrated into the intercalator (EtBr and TMPyP4) solution. Significant hypochromic and bathochromic shifts were observed, confirming the intercalative binding mode (Figure 4a). Two isosbestic points (at 374 and $512 \mathrm{~nm}$ ) were clearly observed for EtBr, which validated the classification of the molecules into either free or bound species. The observed spectra should be the superposition of the absorbance from both species, and the spectral change should be proportional to the percentage of the bound species.

Figure $4 \mathrm{~b}$ shows the spectral change as a function of added DNA concentration. The observed trend can be well-fitted with a noncooperative binding model (see Supporting Information for details). ${ }^{44}$ Through nonlinear least-squares fit, we extracted binding parameters including dissociation constant $\left(K_{\mathrm{d}}\right)$ and saturation binding density $(\Phi)$. Similar extraction was also performed for TMPyP4 (Figure S16). The extracted values displayed in Figure $4 \mathrm{~b}$ and Figure S16b are consistent with previous reports ${ }^{45,46}$ and were used to back-calculate the intercalation density as a function of intercalator and origami concentrations. Figure $4 \mathrm{c}$ presents the relevant calculation for EtBr. Three curves were calculated, corresponding to the three origami concentrations used in our experiments. In all cases, intercalation density initially increases linearly with intercalator concentration and then levels off as the binding density approaches the saturation value. In our experiments, the binding densities were typically limited to less than 0.1 molecule/bp because only moderate binding density was needed for changing origami conformation and excessive intercalation can compromise structural integrity (Figure S17). Within this range (highlighted as blue shade in Figure $4 c)$, the binding density is approximately proportional to binder concentration. This explains the linear relationship between intercalator concentration and the magnitude of induced conformation change.

The binding isotherm can be used to quantitatively predict the required intercalator concentration for inducing a desired amount of conformation change. EtBr unwinds DNA double helices by $26^{\circ}$ per intercalation. ${ }^{29,47}$ To compensate the helicity mismatch in the origami shaft and ribbon $\left(\alpha=17^{\circ}\right.$ in both cases), the required intercalation density should be $\left(17^{\circ} / 32\right.$ bp) $/ 26^{\circ}=1 / 48 \mathrm{bp}^{-1}$. As highlighted by the gray circle in the inset of Figure $4 \mathrm{c}$, the $\mathrm{EtBr}$ concentrations for compensating 1 $\mathrm{nM}$ origami shafts and $2 \mathrm{nM}$ ribbons are predicted to be 0.6 and $0.75 \mu \mathrm{M}$, respectively. The compensating effects (i.e., flattened ribbon and abundant cis shaft) were indeed observed at the concentration range. Such quantitative agreement further 

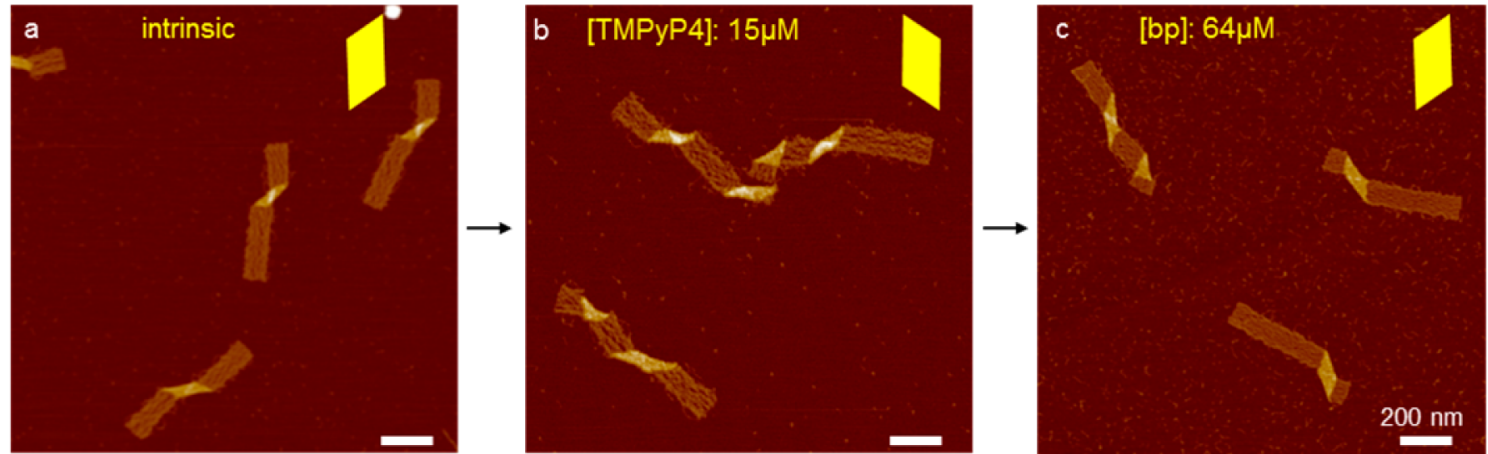

Figure 5. Reversible reconfiguration. AFM images of 8-mer ribbons under intrinsic conditions (a), after addition of $15 \mu \mathrm{M}$ TMPyP4 (b), and after addition of excess DNA strands (corresponding to $64 \mu \mathrm{M}$ base pairs). (c) Ribbons were changed into left-handed twists by TMPyP4 and were then reverted into right-handed twists by adding excess ss-DNA.

validates the molecular mechanism of origami reconfiguration described in Figure 1.

The ability to change DNA origami conformation is not limited to $\mathrm{EtBr}$ and $\mathrm{TMPyP} 4$. Other intercalators (e.g., chloroquine) can induce a similar effect (Figure S18). Nonintercalative DNA-binding molecules may also be incorporated in our reconfiguration scheme. With their ability to induce extra bending and kinks in double-helical DNA, ${ }^{48}$ their conformational effect can be quite distinct. For example, 4',6diamidino-2-phenylindole, a minor groove binder, behaves very differently from intercalators. Instead of inducing flattening, it monotonically increases the twist density in origami ribbons and very effectively rolls them into closed tubular forms (Figure S19).

Reversal of the Conformation Change. In this study, the reconfiguration is induced by the noncovalent binding between intercalator and DNA. The disruption of such binding can reverse the conformation change and is achieved by introducing chemicals that compete with origami in binding with intercalators. Figure 5a,b shows 8-mer ribbons under intrinsic conditions and overcompensated by TMPyP4. By adding excess short DNA strands ( 5 times the mass of the origami in solution), the ribbons were changed back into the original right-handed spiral with a similar degree of twist (Figure 5c). The added DNA strands have similar binding affinity to TMPyP4 as DNA origami. ${ }^{49}$ Their presence causes the redistribution of the adducts and effectively reverses the conformation change. Similarly, reversibility can also be demonstrated with $\mathrm{EtBr}$ (Figure S15), although it is not as efficient as TMPyP4. The kinetics of the reversal process were quite fast, as it typically completed within $10 \mathrm{~min}$. This example demonstrates the reversible nature of intercalator binding and offers a convenient method for reversing the conformation change. Multiple cycles of reconfiguration should be achievable by functionalizing the added DNA strands with collection tags or enclosing them in semipermeable membranes to facilitate clearance of chemical waste.

\section{CONCLUSIONS}

In closing, we have quantitatively studied the ability of DNAbinding adducts to induce conformation change in DNA origami. The reconfiguration process was found to be kinetically fast, linearly controllable, and reversible. The wellconstrained deformation mode, combined with the linear controllability, makes origami shafts promising components for programming nanomechanical motions. By replacing the two flags with two rigid rods, the torsional deformation could be translated into relative circular displacements. More complex movement may be achievable by integrating several shafts with carefully designed length and cross section.

Our reconfiguration mechanism is promising for the ondemand reorganization of functional materials (e.g., enzymes and nanoparticles). It complements the existing reconfiguration strategies such as strand displacement, especially when gradual fine-tuning is more important than complex logical controls. By attaching functional components onto a DNA origami template, the relative distance and orientation between them may be progressively modulated, thereby changing their collective behaviors, such as enzyme cascade efficiency ${ }^{11}$ and plasmonic coupling. ${ }^{21,24}$

This approach is experimentally simple and very adaptable since the DNA origami does not need any chemical modifications, and adducts can be potentially selected from a large pool of DNA-binding molecules (not limited to intercalators) to meet specific application requirements. With the well-understood controllability and the versatile choice of chemical adducts, the mechanism presented in this work should be valuable for dynamic DNA origami and structural DNA technology, in general.

\section{METHODS}

Materials. EtBr was purchased from Bio-Rad. All DNA staples were obtained from IDT (sequence information is in the Supporting Information), and the m13mp18 scaffold was purchased from Bayou Biolabs. All other chemicals were purchased from Sigma-Aldrich.

DNA Origami Preparation. Monomer DNA rectangles and shafts were synthesized by mixing $10 \mathrm{nM} \mathrm{m} 13 \mathrm{mp} 18$ scaffold with $3.5 \times$ staple DNA in $1 \times$ TAE buffer that also contains $12.5 \mathrm{mM}$ magnesium acetate (termed TAEM buffer). The mixture was then thermally annealed from 75 to $4{ }^{\circ} \mathrm{C}$ at $1{ }^{\circ} \mathrm{C} / \mathrm{min}$. To form elongated ribbons, $50 \mathrm{nM}$ of each linker strand in the set was added to the $10 \mathrm{nM}$ monomeric origami tile solution before incubation at $40^{\circ} \mathrm{C}$ for $1 \mathrm{~h}$. Preparation of size-controlled 8-mer ribbons and origami tile cyclization studies are detailed in the Supporting Information.

AFM Imaging. AFM imaging was primarily performed in air to avoid any concentration change caused by evaporation (liquid-phase AFM observed the same features). Before deposition, the origami samples were diluted to the designated concentrations $(0.5,1$, or 2 $\mathrm{nM})$ in a buffer (termed imaging buffer) containing $50 \mathrm{mM} \mathrm{2-}(\mathrm{N}$ morpholino)ethanesulfonic acid, $5 \mathrm{mM}$ magnesium acetate, and 200 $\mathrm{mM} \mathrm{NaCl}$ ( $\mathrm{pH} \sim 6.5$ ) and mixed with varying amounts of intercalators. For deposition, a $20 \mu \mathrm{L}$ sample was pipetted onto freshly cleaved mica and incubated for $\sim 10 \mathrm{~min}$ at room temperature in a closed Petri dish. Compressed air was then used to blow away the sample solution. The dried mica surface was then incubated with $20 \mu \mathrm{L}$ of TAEM buffer for 
1 min before being blown dry and rinsed with $80 \mu \mathrm{L}$ of deionized water. For each statistical data point, at least three $5 \mu \mathrm{m} \times 5 \mu \mathrm{m}$ AFM images were taken from different locations on the mica surface, and at least 200 structures were counted.

Binding Isotherm Titration. The absorption spectra were measured with a PerkinElmer Lambda 950 spectrophotometer. To perform the titration experiment, $800 \mu \mathrm{L}$ binder solutions of suitable concentrations (20 $\mu \mathrm{M}$ for EtBr and $3 \mu \mathrm{M}$ for TMPyP4) in imaging buffer were first measured in a quartz cuvette. The origami tile monomers were previously purified from excess staples by polyethylene glycol coprecipitation ${ }^{50}$ and diluted into suitable concentrations. Mixtures of origami rectangles and adducts were titrated into the solution, while the total binder concentration was kept constant. The mixtures were added in $2 \mu \mathrm{L}$ increments followed by sufficient mixing. The absorption spectrum was recorded after each addition step.

Binding Model. Intercalator-DNA binding was modeled as the following bimolecular reaction: $F+U \rightleftharpoons B O$, where $F$ and $U$ denote free intercalator and unoccupied binding sites on DNA, respectively. $B$ is the bound intercalator, and $O$ represents the occupied binding site; thus $B$ and $O$ appear in pairs. Assuming no binding cooperativity, the binding equilibrium can be expressed as

$$
K_{\mathrm{d}}=\frac{[F][U]}{[B O]}=\frac{[F][U]}{[B]}
$$

where $K_{\mathrm{d}}$ is the dissociation constant. On the basis of linear superposition, the measured absorbance $A$ is

$$
A=\varepsilon_{f}[F]+\varepsilon_{\mathrm{b}}[B]
$$

Here, $\varepsilon_{\mathrm{f}}$ and $\varepsilon_{\mathrm{b}}$ are the molar extinction coefficients of free and bound species, respectively. The total amounts of intercalator and DNA are both known and denoted, respectively, by $C_{\mathrm{I}}$ and $C_{\mathrm{D}}$ (described in the concentration of base pairs). Therefore

$$
\begin{aligned}
& {[F]+[B]=C_{I}} \\
& {[U]+[B]=C_{D} \Phi}
\end{aligned}
$$

where $\Phi$ represents the binding site density on DNA (equals the maximum binding density). The following function can be derived and compared with experimental data.

$$
C_{\mathrm{D}}=\left\{\frac{\varepsilon_{\mathrm{f}} C_{\mathrm{I}}-A}{\varepsilon_{\mathrm{f}}-\varepsilon_{\mathrm{b}}}+K_{\mathrm{d}} \frac{\varepsilon_{\mathrm{f}} C_{\mathrm{I}}-A}{A-\varepsilon_{\mathrm{b}} C_{\mathrm{I}}}\right\} / \Phi
$$

Nonlinear least-squares fit was performed to extract the four floating variables: $K_{\mathrm{d}}, \Phi, \varepsilon_{\mathrm{f}}$ and $\varepsilon_{\mathrm{b}}$. It is notable that $\varepsilon_{\mathrm{f}}$ and $\varepsilon_{\mathrm{b}}$ may also be determined directly from the measured absorbance at $C_{\mathrm{D}}=0$ and $C_{\mathrm{D}}$ $\rightarrow \infty$; the directly measured values are nearly identical to the extracted values.

\section{ASSOCIATED CONTENT}

\section{S Supporting Information}

The Supporting Information is available free of charge on the ACS Publications website at DOI: 10.1021/acsnano.6b01339.

Additional analysis, supporting figures, design details, and sequence information (PDF)

\section{AUTHOR INFORMATION}

\section{Corresponding Author}

*E-mail: jchoi@purdue.edu.

\section{Notes}

The authors declare no competing financial interest.

\section{ACKNOWLEDGMENTS}

This work was supported by the U.S. National Science Foundation (Grant Nos. 1055866, 1437301, and 1512537) and Office of Naval Research (N00014-15-1-2707). The funding from the Swedish Research Council VR is also gratefully acknowledged.

\section{REFERENCES}

(1) Seeman, N. C. DNA in a Material World. Nature 2003, 421, 427-431

(2) Williamson, J. R. RNA Origami. Nat. Struct. Biol. 1994, 1, 270272

(3) Seeman, N. C. De Novo Design of Sequences for Nucleic Acid Structural Engineering. J. Biomol. Struct. Dyn. 1990, 8, 573-581.

(4) Tumpane, J.; Kumar, R.; Lundberg, E. P.; Sandin, P.; Gale, N.; Nandhakumar, I. S.; Albinsson, B.; Lincoln, P.; Wilhelmsson, L. M.; Brown, T.; et al. Triplex Addressability as a Basis for Functional DNA Nanostructures. Nano Lett. 2007, 7, 3832-3839.

(5) Tumpane, J.; Sandin, P.; Kumar, R.; Powers, V. E.; Lundberg, E. P.; Gale, N.; Baglioni, P.; Lehn, J.-M.; Albinsson, B.; Lincoln, P.; et al. Addressable High-Information-Density DNA Nanostructures. Chem. Phys. Lett. 2007, 440, 125-129.

(6) Rothemund, P. W. K. Folding DNA to Create Nanoscale Shapes and Patterns. Nature 2006, 440, 297-302.

(7) Wang, Z.-G.; Liu, Q.; Ding, B. Shape-Controlled Nanofabrication of Conducting Polymer on Planar DNA Templates. Chem. Mater. 2014, 26, 3364-3367.

(8) Steinhauer, C.; Jungmann, R; Sobey, T. L.; Simmel, F. C.; Tinnefeld, P. DNA Origami as a Nanoscopic Ruler for SuperResolution Microscopy. Angew. Chem., Int. Ed. 2009, 48, 8870-8873.

(9) Fu, J.; Liu, M.; Liu, Y.; Woodbury, N. W.; Yan, H. Interenzyme Substrate Diffusion for an Enzyme Cascade Organized on Spatially Addressable DNA Nanostructures. J. Am. Chem. Soc. 2012, 134, 55165519.

(10) Fu, Y.; Zeng, D.; Chao, J.; Jin, Y.; Zhang, Z.; Liu, H.; Li, D.; Ma, H.; Huang, Q.; Gothelf, K. V.; et al. Single-Step Rapid Assembly of DNA Origami Nanostructures for Addressable Nanoscale Bioreactors. J. Am. Chem. Soc. 2013, 135, 696-702.

(11) Linko, V.; Eerikäinen, M.; Kostiainen, M. A. A Modular DNA Origami-Based Enzyme Cascade Nanoreactor. Chem. Commun. 2015, 51, 5351-5354.

(12) Kuzyk, A.; Schreiber, R.; Fan, Z.; Pardatscher, G.; Roller, E.-M.; Högele, A.; Simmel, F. C.; Govorov, A. O.; Liedl, T. DNA-Based SelfAssembly of Chiral Plasmonic Nanostructures with Tailored Optical Response. Nature 2012, 483, 311-314.

(13) Pilo-Pais, M.; Watson, A.; Demers, S.; LaBean, T. H.; Finkelstein, G. Surface-Enhanced Raman Scattering Plasmonic Enhancement Using DNA Origami-Based Complex Metallic Nanostructures. Nano Lett. 2014, 14, 2099-2104.

(14) Surwade, S. P.; Zhao, S.; Liu, H. Molecular Lithography through DNA-Mediated Etching and Masking of $\mathrm{SiO}_{2}$. J. Am. Chem. Soc. 2011, 133, 11868-11871.

(15) Surwade, S. P.; Zhou, F.; Wei, B.; Sun, W.; Powell, A.; O'Donnell, C.; Yin, P.; Liu, H. Nanoscale Growth and Patterning of Inorganic Oxides Using DNA Nanostructure Templates. J. Am. Chem. Soc. 2013, 135, 6778-6781.

(16) Jin, Z.; Sun, W.; Ke, Y.; Shih, C.-J.; Paulus, G. L.; Wang, Q. H.; Mu, B.; Yin, P.; Strano, M. S. Metallized DNA Nanolithography for Encoding and Transferring Spatial Information for Graphene Patterning. Nat. Commun. 2013, 4, 1663.

(17) Choi, J.; Chen, H.; Li, F.; Yang, L.; Kim, S. S.; Naik, R. R.; Ye, P. D.; Choi, J. H. Nanomanufacturing of 2d Transition Metal Dichalcogenide Materials Using Self-Assembled DNA Nanotubes. Small 2015, 11, 5520-5527.

(18) Kuzuya, A.; Sakai, Y.; Yamazaki, T.; Xu, Y.; Komiyama, M. Nanomechanical DNA Origami 'Single-Molecule Beacons' Directly Imaged by Atomic Force Microscopy. Nat. Commun. 2011, 2, 449.

(19) Douglas, S. M.; Bachelet, I.; Church, G. M. A Logic-Gated Nanorobot for Targeted Transport of Molecular Payloads. Science 2012, 335, 831-834. 
(20) Andersen, E. S.; Dong, M.; Nielsen, M. M.; Jahn, K.; Subramani, R.; Mamdouh, W.; Golas, M. M.; Sander, B.; Stark, H.; Oliveira, C. L.; et al. Self-Assembly of a Nanoscale DNA Box with a Controllable Lid. Nature 2009, 459, 73-76.

(21) Kuzyk, A.; Schreiber, R.; Zhang, H.; Govorov, A. O.; Liedl, T.; Liu, N. Reconfigurable 3D Plasmonic Metamolecules. Nat. Mater. 2014, 13, 862-866.

(22) Zhang, D. Y.; Seelig, G. Dynamic DNA Nanotechnology Using Strand-Displacement Reactions. Nat. Chem. 2011, 3, 103-113.

(23) Yang, Y. Y.; Endo, M.; Hidaka, K.; Sugiyama, H. PhotoControllable DNA Origami Nanostructures Assembling into Predesigned Multiorientational Patterns. J. Am. Chem. Soc. 2012, 134, 20645-20653.

(24) Kuzyk, A.; Yang, Y.; Duan, X.; Stoll, S.; Govorov, A. O.; Sugiyama, H.; Endo, M.; Liu, N. A Light-Driven Three-Dimensional Plasmonic Nanosystem That Translates Molecular Motion into Reversible Chiroptical Function. Nat. Commun. 2016, 7, 10591.

(25) Gerling, T.; Wagenbauer, K. F.; Neuner, A. M.; Dietz, H. Dynamic DNA Devices and Assemblies Formed by ShapeComplementary, Non-Base Pairing 3D Components. Science 2015, $347,1446-1452$.

(26) Bustamante, C.; Bryant, Z.; Smith, S. B. Ten Years of Tension: Single-Molecule DNA Mechanics. Nature 2003, 421, 423-427.

(27) Kauert, D. J.; Kurth, T.; Liedl, T.; Seidel, R. Direct Mechanical Measurements Reveal the Material Properties of Three-Dimensional DNA Origami. Nano Lett. 2011, 11, 5558-5563.

(28) Zhou, L.; Marras, A. E.; Su, H.-J.; Castro, C. E. DNA Origami Compliant Nanostructures with Tunable Mechanical Properties. ACS Nano 2014, 8, 27-34.

(29) Zeman, S. M.; Depew, K. M.; Danishefsky, S. J.; Crothers, D. M. Simultaneous Determination of Helical Unwinding Angles and Intrinsic Association Constants in Ligand-DNA Complexes: The Interaction between DNA and Calichearubicin B. Proc. Natl. Acad. Sci. U. S. A. 1998, 95, 4327-4332.

(30) Ke, Y.; Bellot, G.; Voigt, N. V.; Fradkov, E.; Shih, W. M. Two Design Strategies for Enhancement of Multilayer-DNA-Origami Folding: Underwinding for Specific Intercalator Rescue and StapleBreak Positioning. Chem. Sci. 2012, 3, 2587-2597.

(31) Zhao, Y.-X.; Shaw, A.; Zeng, X.; Benson, E.; Nystrom, A. M.; Hogberg, B. DNA Origami Delivery System for Cancer Therapy with Tunable Release Properties. ACS Nano 2012, 6, 8684-8691.

(32) Dietz, H.; Douglas, S. M.; Shih, W. M. Folding DNA into Twisted and Curved Nanoscale Shapes. Science 2009, 325, 725-730.

(33) Woo, S.; Rothemund, P. W. Programmable Molecular Recognition Based on the Geometry of DNA Nanostructures. Nat. Chem. 2011, 3, 620-627.

(34) Castro, C. E.; Kilchherr, F.; Kim, D.-N.; Shiao, E. L.; Wauer, T.; Wortmann, P.; Bathe, M.; Dietz, H. A Primer to Scaffolded DNA Origami. Nat. Methods 2011, 8, 221-229.

(35) Jungmann, R.; Scheible, M.; Kuzyk, A.; Pardatscher, G.; Castro, C. E.; Simmel, F. C. DNA Origami-Based Nanoribbons: Assembly, Length Distribution, and Twist. Nanotechnology 2011, 22, 275301.

(36) Li, Z.; Wang, L.; Yan, H.; Liu, Y. Effect of DNA Hairpin Loops on the Twist of Planar DNA Origami Tiles. Langmuir 2012, 28, 19591965.

(37) Chen, H. R.; Weng, T. W.; Riccitelli, M. M.; Cui, Y.; Irudayaraj, J.; Choi, J. H. Understanding the Mechanical Properties of DNA Origami Tiles and Controlling the Kinetics of Their Folding and Unfolding Reconfiguration. J. Am. Chem. Soc. 2014, 136, 6995-7005.

(38) Shore, D.; Langowski, J.; Baldwin, R. L. DNA Flexibility Studied by Covalent Closure of Short Fragments into Circles. Proc. Natl. Acad. Sci. U. S. A. 1981, 78, 4833-4837.

(39) Rocha, M.; Ferreira, M.; Mesquita, O. Transition on the Entropic Elasticity of DNA Induced by Intercalating Molecules. J. Chem. Phys. 2007, 127, 105108.

(40) Kaji, N.; Ueda, M.; Baba, Y. Direct Measurement of Conformational Changes on DNA Molecule Intercalating with a Fluorescence Dye in an Electrophoretic Buffer Solution by Means of Atomic Force Microscopy. Electrophoresis 2001, 22, 3357-3364.
(41) Kim, D.-N.; Kilchherr, F.; Dietz, H.; Bathe, M. Quantitative Prediction of 3D Solution Shape and Flexibility of Nucleic Acid Nanostructures. Nucleic Acids Res. 2012, 40, 2862-2868.

(42) Ke, Y.; Douglas, S. M.; Liu, M.; Sharma, J.; Cheng, A.; Leung, A.; Liu, Y.; Shih, W. M.; Yan, H. Multilayer DNA Origami Packed on a Square Lattice. J. Am. Chem. Soc. 2009, 131, 15903-15908.

(43) Paul, A.; Bhattacharya, S. Chemistry and Biology of DNABinding Small Molecules. Curr. Sci. 2012, 102, 212-231.

(44) Chaires, J. B.; Dattagupta, N.; Crothers, D. M. Studies on Interaction of Anthracycline Antibiotics and Deoxyribonucleic Acid: Equilibrium Binding Studies on the Interaction of Daunomycin with Deoxyribonucleic Acid. Biochemistry 1982, 21, 3933-3940.

(45) Garbett, N. C.; Hammond, N. B.; Graves, D. E. Influence of the Amino Substituents in the Interaction of Ethidium Bromide with DNA. Biophys. J. 2004, 87, 3974-3981.

(46) Fiel, R.; Munson, B. Binding of Meso-Tetra (4-N-Methylpyridyl) Porphine to DNA. Nucleic Acids Res. 1980, 8, 2835-2842.

(47) Liu, L. F.; Wang, J. C. On the Degree of Unwinding of the DNA Helix by Ethidium: II. Studies by Electron Microscopy. Biochim. Biophys. Acta, Nucleic Acids Protein Synth. 1975, 395, 405-412.

(48) Zimmer, C.; Wähnert, U. Nonintercalating DNA-Binding Ligands: Specificity of the Interaction and Their Use as Tools in Biophysical, Biochemical and Biological Investigations of the Genetic Material. Prog. Biophys. Mol. Biol. 1986, 47, 31-112.

(49) Ren, J.; Chaires, J. B. Sequence and Structural Selectivity of Nucleic Acid Binding Ligands. Biochemistry 1999, 38, 16067-16075.

(50) Stahl, E.; Martin, T. G.; Praetorius, F.; Dietz, H. Facile and Scalable Preparation of Pure and Dense DNA Origami Solutions. Angew. Chem., Int. Ed. 2014, 53, 12735-12740. 